\title{
An Optimized Field Function Scheme for Nanoparticle Guidance in Magnetic Drug Targeting systems
}

\author{
Ton Duc Do, member, IEEE, Yeongil Noh, Myeong Ok Kim, and Jungwon Yoon, member, IEEE
}

\begin{abstract}
Magnetic drug targeting is an approach to guide and concentrate magnetic nanoparticles (MNPs) into the diseased target organ after being injected into blood vessels. Although many works for drug targeting have been conducted, there are few studies on delivering the nanoparticles to the target region. Drug delivery performance has not been addressed sufficiently or fully. In this paper, we investigate the effect of dominant factors to MNPs delivery performance. Then, an optimized field function scheme with a pulsed magnetic actuation is proposed to significantly improve the MNPs guidance performance. With a specific condition of blood vessel size, particle size, and applied magnetic field, the optimized parameters of the field function are selected through extensive simulation studies. We find out that the optimal negative and positive time for the magnetic pulsed field mainly depends on the exit time for particles to reach the bifurcation and the critical time as the maximum time for them to reach the vessels wall, respectively. With the chosen parameters, we show that ratios of correctly guided particles in a Y-channel are reached to $100 \%$. In addition, to minimize the power consumption, a modified field function (MFF) scheme is introduced. The MFF includes a no-power time, called zero-time, between the positive and negative time. It is shown that with the proposed MFF, the energy consumption and the heating problem of the actuator system can be significantly reduced. Therefore, the proposed guidance scheme for MNPs can overcome the sticking issue and maximize the guidance performance as well as reducing the power consumption. It should be noted that the MFF can be easily implement by programmable DC power supplies connected to electromagnetic coils.
\end{abstract}

Index Terms-Drug delivery, Field function, Magnetic drug targeting, Magnetic nanoparticle, Optimization.

This research was supported by the Pioneer Research Center Program through the National Research Foundation of Korea funded by the Ministry of Science, ICT \& Future Planning (2012-0009524).

T. D. Do is with the School of Mechanical and Aerospace Engineering and Pioneer Research Center for Controlling Dementia by Converging Technology, Gyeongsang National University, Jinju, 660-701, Republic of Korea (e-mail: tonducdo@gnu.ac.kr)

Y. Noh and J. Yoon are with the School of Mechanical and Aerospace Engineering, Gyeongsang National University, Jinju 660-701, Republic of Korea (e-mail: jwyoon@gnu.ac.kr).

M. O. Kim is with the Department of Biology and Applied of Life Science, Gyeongsang National University, Jinju, 660-701, Republic of Korea (e-mail: mokim@gnu.ac.kr).

\section{INTRODUCTION}

Magnetic drug delivery or magnetic drug targeting (MDT) is a technique that adds drugs to magnetic nano/micro-size particles and then applies external magnetic fields to steer and concentrate them at disease locations, such as solid tumors, regions of infection, or blood clots [1], [2]. The drug dosage and administration rate should result in temporal and spatial drug concentrations that are below the toxic level and above the level of minimal therapeutic effects. The normal circulation of the blood will distribute the drug throughout the body and so systemic toxicity may occur in organs to which the drug is not targeted. Magnetic drug targeting allows the drug dosage to be increased significantly while avoiding side effects in healthy tissues [3], [4].

Recently, there are a lot of researches on MDT which focus on the capture and retention of magnetic nanoparticles [5]-[9]. The retention of superparamagnetic nanoparticles under the influence of a high-gradient magnetic field was investigated by Gleich [5]. Cherry [6] developed a physics-based model of a general magnetic drug targeting system to determine the effect of drug particle size, blood flow velocity, and magnetic field gradient strength on the efficiency of holding particles stationary in a laminar blood flow in a medium-sized artery. It was found that particle retention rate increased. David et al. presented a combined theoretical and in vitro modeling approach for predicting the magnetic capture and retention of magnetic nanoparticles in vivo [7]. Furthermore, the behaviors of magnetic particles in blood vessels and surrounding tissues were analyzed comprehensively, using physical first-principles and a sophisticated vessel-membrane-tissue (VMT) numerical solver in [8], and simulations of magnetic capturing of drug carriers in the brain vascular system in [9].

Recently, some researchers have attempted to investigate nanoparticle behavior and possible issues during the guiding phase inside the blood vessel [10]-[12]. In [13], we showed that the sticking of particles to vessel walls is an issue in the transport of the particles towards the target region. Although adherence of the particles to walls is the main goal in the target region and capturing phase, it is not desirable in the delivery stage and leads to low transport efficiency. In [10], 
the sticking and concentration of particles near a Y-shape channel was reported. Likewise, in [11] and [12], the adherence of nanoparticles to vessels with a constant magnetic field was examined experimentally and through simulation, respectively. In [13], although sticking was reduced slightly by changing the shape of the magnet, this is not a general solution because the study focused only on the special case of particles sticking at the injection site. In [12], the steering efficiency in the special case of a 2D Y shape channel was increased using the aggregation properties of the particles and a special magnetic field. The authors claimed that the best steering strategy was to generate aggregations, the angle of attack of which was orthogonal to the fluid flow. However, aggregations orthogonal to the flow may result in clogging of the branches, which is clearly not desirable in drug delivery to brain. To date, sticking of magnetic nanoparticles in blood vessels and the effects on steering efficiency has not been investigated. In [14], the authors suggested the concept of a functionalized magnetic field, named field function, which generates a simple direction-varying magnetic gradient instead of a constant-direction magnetic gradient to solve the particle sticking to the vessel walls problem. Numerous simulation results were shown to prove the field function concept. Even though the performance improvement by the field function compared to a constant magnetic field had been efficiently shown in the previous research, it was difficult to find adequate field function parameters with different guidance conditions.

In this paper, the delivery performance of magnetic nanoparticles with a field function is maximized systematically using numerical simulations via proposing optimization conditions. Moreover, a modified field function scheme is suggested to further improve the working conditions of the actuator systems. The functionalities and capabilities of this approach were evaluated in detail with several numerical simulations. The outcomes show the effectiveness of the proposed field function scheme for targeted drug delivery, which can maximize the MNPs delivery performance as well as significantly reducing power consumption and heating problems.

\section{GUIDANCE SCHEME AND FIELD FUNCTIONS}

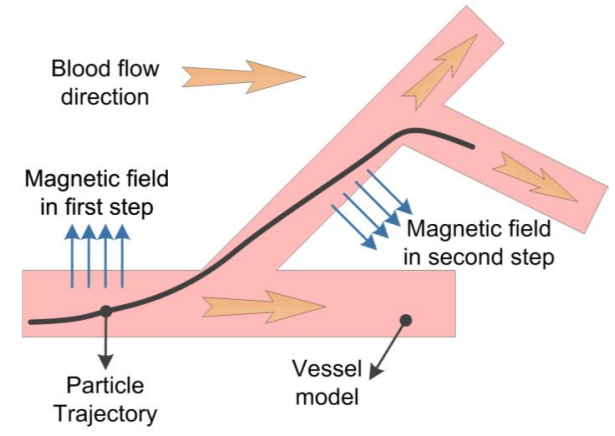

Fig. 1 The MNPs' steering approach
In our guiding method for MNPs, the blood flow propels the particles and the electromagnets are used only for steering, by simply altering the radial position of the particles moving with the blood inside the vessels to direct them to the desired exit at the next bifurcation. If the particles are positioned in the appropriate region of the vessel before arriving at the bifurcation, they will enter the correct branch. Fig. 1 shows a simple model of this guidance approach [11]. Due to the very light weight of the nanoparticles, the blood can carry them through $10 \mu \mathrm{m}$ diameter capillaries and the electromagnetic actuation system simply moves the particles in a direction that is perpendicular to the blood flow inside the vessel. The drag force exerted on a particle in transition perpendicular to the blood flow is less than the drag force in the flow direction. As a result, the magnetic force needed to overcome the drag force for steering is much less than the force needed for propulsion.

However, the magnetic particle guidance inside the blood vessel by a constant-direction magnetic field is not efficient, due to particles' sticking to the walls. If the particles can be returned to the central region of the vessel, the sticking number of the particles can be decreased and the number of particles reaching to the correct outlet can increase. The field function $(\mathrm{FF})$ is a unitless multiplier function that varies by time, which defines how to change the magnetic field gradient over time with the following constraints:

$$
\forall t-1 \leq F F(t) \leq 1
$$

If we suppose that $\nabla \boldsymbol{H}_{f}$ is a functionalized magnetic field gradient, we have:

$$
\nabla \mathbf{H}_{f}=F F(t) . \nabla \mathbf{H}
$$

Here, $\nabla \boldsymbol{H}$ is the predefined constant magnetic field, designed to direct the particle to the correct outlet, and therefore, its direction does not change. This definition implies that the functionalized gradient is always parallel to the predefined gradient field and the FF just can change its magnitude and sign. The functionalized force on the particles is defined as follows:

$$
\boldsymbol{F}_{f}=\frac{4}{3} \pi \mu_{1} R^{3} \boldsymbol{M}_{\text {sat }} . \nabla \boldsymbol{H}_{f}=F F(t)\left[\frac{4}{3} \pi \mu_{1} R^{3} \boldsymbol{M}_{\text {sat }} . \nabla \boldsymbol{H}\right]
$$

where $R$ and $M_{\text {sat }}$ are the particles radius and saturation value of net magnetic polarization, respectively. Consequently, FF can be used as a simple scalar function that defines the force magnitude and direction in a more applicable way. When the FF is positive, this means that the force applied is in a direction that directs particles to the correct outlet and when it is negative, the force applied guides particles to the incorrect outlet. A simple rectangular function was used for the FF. The general characteristics of the FFs are shown in Fig.2.

In this figure, $T_{f}$ is the total simulation time that was considered for steering. If we assume $D_{c}$ is the number of cycles in the simulation time, the period $(P)$, and frequency $(f)$ of the FF can be calculated

$$
P=\frac{1}{f}=\frac{T_{f}}{N_{c}}
$$

Duty cycle is another parameter of the FF, defined as $0 \leq$ $D_{c} \leq 1$. By this definition, the portion of the period in which 
the function is positive in relation to the portion in which the function is negative equals to $D_{c} /\left(1-D_{c}\right)$.

Although the duty cycle of the function may affect the guidance results, the duty cycle was fixed to 0.7 [12] for simulation simplicity and the period of the FF was discretely selected by trial and errors. Therefore, it is necessary to optimize parameters of the field function for better guidance performance.

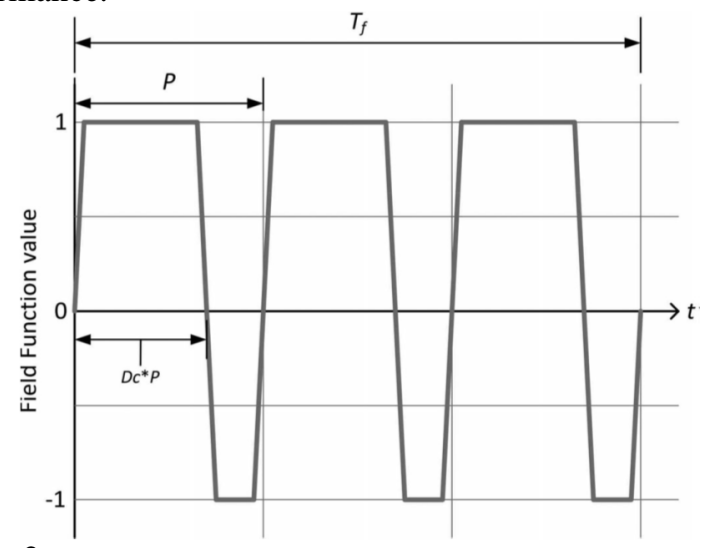

Fig. 2 Characteristics of the original FF.

\section{OPTIMIZING THE PARAMETERS OF FIELD FUNCTION}

This section focuses on how to choose the optimized parameters of the field function to improve the MNPs delivery performance. Through analyses and extensive simulation studies, the optimal parameters of field function are suggested.

\section{A. Definitions}

\section{1) Field function parameters}

In one period, we define the time period that the field function is positive is $T_{\text {plus }}$, and the time period that the field function is negative is $\mathrm{T}_{\text {minus }}$. The waveform of the field function is shown in Fig. 3.

\section{2) Safe zone}

Safe zone is a part of the vessel that all the particles inside this zone will reach to a correct outlet even when there are no external forces applied to the particles. Therefore, we need to steer all the particles to the safe zone. Fig. 4 demonstrates the safe zone in a Y-shape channel.

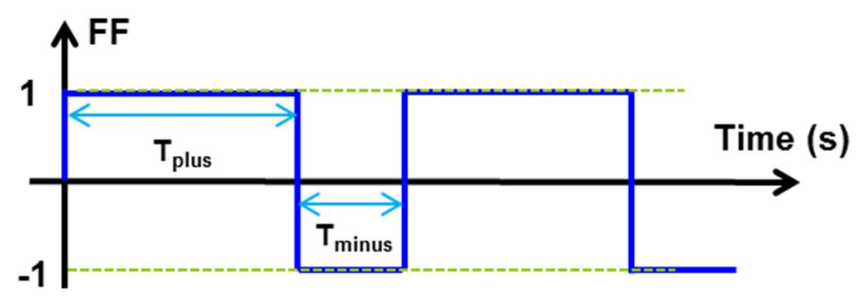

Fig. 3. Waveform of the field function.

\section{3) Exit time}

The exit time is defined as a time for a particle at the center of the vessel to reach horizontally from the staring point to the bifurcation:

$$
T_{x}=\frac{l_{\mathrm{v}}}{v_{p x}^{a}}
$$

where $\mathrm{m}_{\mathrm{p}} l_{v}$ and $v_{p x}^{a} \mathrm{v}_{\mathrm{p}}$ are the length of the vessel and the average velocity in the $\mathrm{x}$-coordinate of particles before reaching to the bifurcation, respectively. Fig. 5 illustrates the exit time in case of a Y-shape vessel.

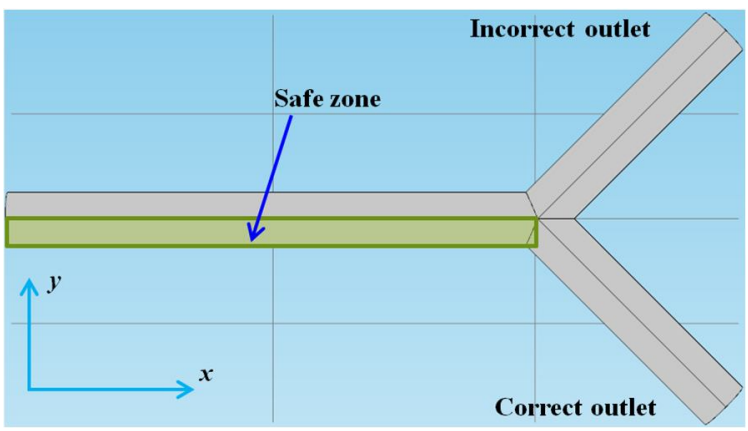

Fig. 4 Demonstration of the safe zone in a Y-shape channel.

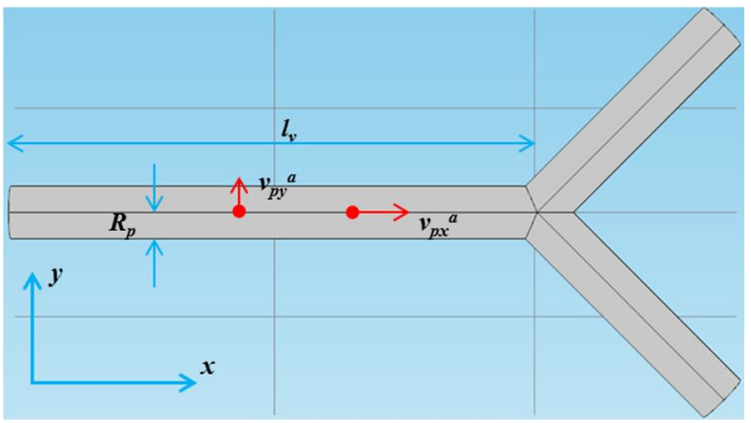

Fig. 5. Demonstration of the exit time and critical time in a Y-shape channel.

\section{4) Critical time}

Critical time is a time that a particle should reach vertically from a wall position to the center of the vessel, and vice versa:

$$
T_{c}=\frac{R_{\mathrm{p}}}{v_{p y}^{a}}
$$

where $R_{p}$ and $v_{p y}^{a} \mathrm{v}_{\mathrm{p}}$ are the radius of the vessel and the average velocity in the $y$-coordinate of particles before reaching to the bifurcation, respectively. Fig. 5 also shows the critical time with a Y-shape vessel.

\section{B. Optimized Conditions}

In this subsection, we propose the best conditions in order to steer all particles to the correct outlet.

Condition 1: All particles must be in the safe zone before the magnetic force changes its direction. This condition can be expressed by the following equation:

$$
T_{\text {plus }} \geq \alpha T_{x}
$$

where $\alpha>1$ is a coefficient considering the effects of multiple particles to the exit time.

Condition 2: The negative time to release the particles sticking at the vessel walls should be smaller than the critical time:

$$
T_{\text {minus }} \leq \beta T_{c}
$$

where $\beta<1$ is a coefficient considering the effect of multiple 
particles to the critical time.

Condition 3: All particles must be in the safe zone before reaching to the bifurcation:

$$
T_{c} \leq T_{x}
$$

The conditions 1 and 2 will be used to select the optimal parameters for the field function. The condition 3 is the necessary condition to steer all the particles to the correct outlet.

The criteria to choose the optimal parameters of FF are as follows:

1) Typically, $T_{\text {plus }}$ is high. Therefore, $T_{\text {plus }}$ should be as small as possible to achieve fast response.

2) Normally, $T_{\text {minus }}$ is much smaller than $T_{\text {plus. }}$. Therefore, $\mathrm{T}_{\text {minus }}$ should be closer to $\mathrm{T}_{\text {plus }}$ and as high as possible to achieve symmetric actuation system, which results in the minimal heating problem.

For the optimal criteria, $\mathrm{T}_{\text {plus }}$ and $\mathrm{T}_{\text {minus }}$ should approach to $\mathrm{T}_{\mathrm{x}}$ and $\mathrm{T}_{\mathrm{c}}$, respectively, as closely as possible with the conditions of $\alpha=\beta=1$, which means the smallest actuation efforts and actuation symmetry with the best performances .

Because it is not easy to calculate the exit time and critical time as well as the coefficient $\alpha$ and $\beta$, numerical studies through simulations are required to estimate these values.

\section{Numerical Simulation Studies}

TABLE I. MODELLING PARAMETERS.

\begin{tabular}{lll}
\hline Parameter & Value & Unit \\
\hline Blood density & 1050 & $\mathrm{~kg} / \mathrm{m}^{3}$ \\
\hline Blood viscosity & 0.004 & $\mathrm{~Pa} . \mathrm{s}$ \\
\hline Air relative permeability & 1 & - \\
\hline Blood relative permeability & 1 & - \\
\hline Blood temperature & 293.15 & $\mathrm{~K}$ \\
\hline Particle diameter & 800 & $\mathrm{Nm}$ \\
\hline particle density & 6450 & $\mathrm{~kg} / \mathrm{m}^{3}$ \\
\hline Blood velocity & 1 & $\mathrm{~mm} / \mathrm{s}$ \\
\hline Vessel length & 10 & $\mathrm{Mm}$ \\
\hline Vessel diameter & 1 & $\mathrm{Mm}$ \\
\hline Magnetic field gradient & 3300 & $\mathrm{kA} / \mathrm{m}^{2}$ \\
\hline Saturation magnetization & 106 & $\mathrm{kA} / \mathrm{m}$ \\
\hline
\end{tabular}

In this paper, a simple Y-shape channel was used as a vessel model for simulation (Figs. 3 and 4). The Y-shape channel consists of one inlet for entering blood flow and particles and two outlet channels. The diameter of the vessel in all paths was assumed to be identical. The blood flow is considered to be steady creeping flow, which was supposed to flow into the channel from the inlet and to exit the channel from the outlets. Fluid modeling parameters were selected to be similar to blood behavior [15] and the surrounding environment was assumed to be air. The blood flow inside the channels was simulated as a steady state laminar flow and its velocity profile was calculated using the CFD module of COMSOL. The blood flow drag force was considered in the simulation. The particles were assumed to be spherical magnetite particles with a diameter of $d_{p}$, a density of $6450 \mathrm{~kg} / \mathrm{m}^{3}$, and saturation magnetization of $\mathbf{M}_{\mathrm{sat}}=106 \mathrm{kA} / \mathrm{m}$, [16]. These modeling parameters as well as other parameters for the model are presented in Table I. The model was created in 3D and meshed automatically.

In order to estimate the exit time and critical time, only one particle is injected at the center of the vessel at the inlet. In case of estimating the exit time, no external magnetic field is applied. Fig. 6 demonstrates the simulation model to estimate the exit and critical times. Through this simulation, the exit time $T_{x}$ is approximately $7.5 \mathrm{~s}$. To estimate the critical time, the magnetic field with the values in Table I is applied to the MNP. Through this simulation, the critical time $T_{c}$ is also 7.5 s. Because the exit time and the critical time are equal, the condition 3 can be satisfied.

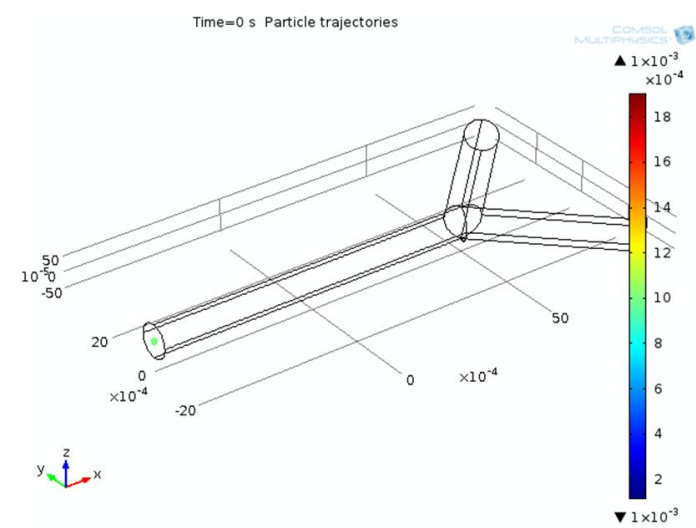

Fig. 6. The model for estimating exit time. The bar indicates the velocity of particles.

TABle II. Simulation Results of the Particle Ratios to the CoRrect OUTLET WITH DIFFERENT VALUES OF $\mathrm{T}_{\text {PLUS }}$ AND $\mathrm{T}_{\text {MINUS }}$

\begin{tabular}{|c|c|c|c|c|c|c|}
$\begin{array}{c}\boldsymbol{T}_{\text {plus }}^{(\mathbf{s})} \\
\boldsymbol{T}_{\text {minus }}(\mathbf{s})\end{array}$ & 7.5 & 8 & 8.5 & 9 & 9.5 & 10 \\
\hline 3 & $99.4 \%$ & $99.9 \%$ & $100 \%$ & $100 \%$ & $100 \%$ & $100 \%$ \\
\hline 3.5 & $99.2 \%$ & $99.9 \%$ & $100 \%$ & $100 \%$ & $100 \%$ & $100 \%$ \\
\hline 4 & $99.2 \%$ & $99.8 \%$ & $100 \%$ & $100 \%$ & $100 \%$ & $100 \%$ \\
\hline 4.5 & $99.2 \%$ & $99.8 \%$ & $\underline{\mathbf{1 0 0 \%}}$ & $100 \%$ & $100 \%$ & $100 \%$ \\
\hline 5 & $99.2 \%$ & $99.8 \%$ & $99.9 \%$ & $100 \%$ & $100 \%$ & $100 \%$ \\
\hline 5.5 & $99.0 \%$ & $99.8 \%$ & $99.9 \%$ & $100 \%$ & $100 \%$ & $100 \%$ \\
\hline 6 & $99.0 \%$ & $99.6 \%$ & $99.8 \%$ & $100 \%$ & $100 \%$ & $100 \%$ \\
\hline 6.5 & $98.5 \%$ & $99.6 \%$ & $99.8 \%$ & $99.9 \%$ & $100 \%$ & $100 \%$ \\
\hline 7 & $98.3 \%$ & $99.1 \%$ & $99.4 \%$ & $99.7 \%$ & $99.8 \%$ & $99.9 \%$ \\
\hline 7.5 & $97.5 \%$ & $98.6 \%$ & $99.0 \%$ & $99.1 \%$ & $99.5 \%$ & $99.6 \%$ \\
\hline
\end{tabular}

Next step is to find out the values of $\alpha$ and $\beta$ for $100 \%$ of particles to reach the correct outlet. The minimum positive time $T_{\text {plus }}$ and the maximum negative time $T_{\text {minus }}$ are $7.5 \mathrm{~s}$. Table II summaries the simulation results of the ratio of the particles that reaches to the correct outlet. Fig. 7.a and Fig. 7.b present the particle trajectory for $\mathrm{T}_{\text {plus }}=\mathrm{T}_{\text {minus }}=7.5 \mathrm{~s}$ and $\mathrm{T}_{\text {plus }}$ $=8.5, \mathrm{~T}_{\text {minus }}=4.5 \mathrm{~s}$, respectively. As shown in the Table II, to achieve the maximum delivery performance, with a specific of positive time (satisfied $T_{\text {plus }} \geq 8.5 \mathrm{~s}$ ), the negative time needs to be smaller than a certain value. For example: If $T_{\text {plus }}=8.5 \mathrm{~s}$ then $T_{\text {minus }} \leq 4.5 \mathrm{~s}(\alpha=1.13$ and $\beta \leq 0.6)$. 
So far, we present the guidelines to choose the optimal value of $\mathrm{T}_{\text {plus }}$ and $\mathrm{T}_{\text {minus }}$ (in other words, duty ratio and frequency), which was not considered in [12]. The minimum value of $T_{\text {plus }}=8.5 \mathrm{~s}$. It means that the criterion 1 has been achieved with this value. Although the value of $\alpha$ is near 1 , the value of $\beta$ is quite far from 1 . In other words, the criteria 2 and 3 are still not minimized. To further optimize the working conditions, it is desirable to increase $\beta$ for being closer to 1 .

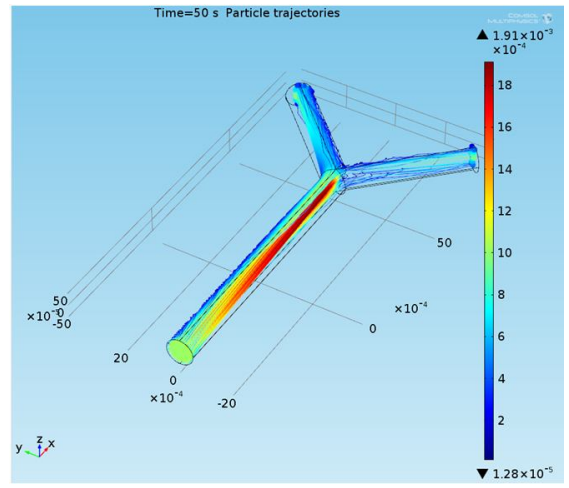

Fig. 7.a. Particle trajectories for 1000 particles with $\mathrm{T}_{\text {plus }}=7.5 \mathrm{~s}, \mathrm{~T}_{\text {minus }}=7.5 \mathrm{~s}$. Colors show the particles velocity $(\mathrm{mm} / \mathrm{s})$.

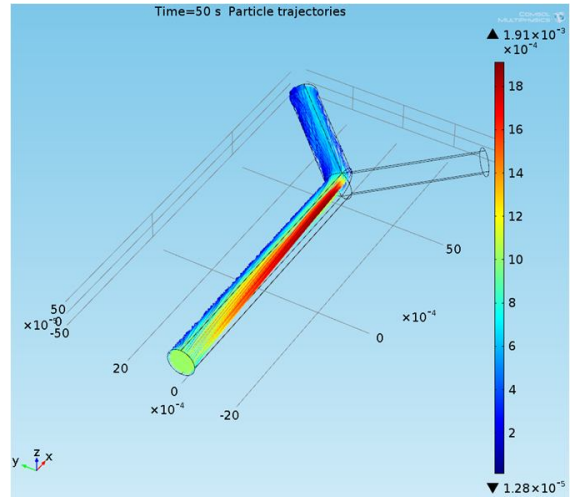

Fig. 7.b. Particle trajectories for 1000 particles with $\mathrm{T}_{\text {plus }}=8.5 \mathrm{~s}, \mathrm{~T}_{\text {minus }}=4.5 \mathrm{~s}$. Colors show the particles velocity $(\mathrm{mm} / \mathrm{s})$.

\section{MOdIFIED FIELD FunCTION}

\section{A. The Concept of Modified Field Function}

In the previous section, we show that by carefully considering the exit time and critical time, we can select the optimal value to maximize the particles' success ratio to the correct outlet. However, the field function concept in the previous section includes the following drawback: When the particles are in the safe zone, even if we do not provide external magnetic force, they will reach to the correct branch (except for particles stuck at the vessel wall). If we immediately change the direction of the magnetic force, some particles near the boundary may not stay in the safe zone. Therefore, it is better if we provide a period with no magnetic force before we apply a negative force. The new field function named as modified field function (MFF) is demonstrated in Fig. 8. The time period during which there is no power is a called zero time $\mathrm{T}_{\text {zero. }}$. The proposed MFF offers the following advantages:

1. Reduce the heating problem of the coils if the electromagnetic actuator is used to produce magnetic field. As the zero time is added in each cycle of the MFF, each coil has more time to rest. Therefore, the temperature of each coil can be reduced further.

2. The power consumption is also reduced. The power consumption is proportional to the time during which the power is on. In case of the field function, the power is on $100 \%$, however, in case of the MFF, the power is on $\frac{\left(T_{\text {plus }}+T_{\text {minus }}\right)}{\left(T_{\text {plus }}+T_{\text {zero }}+T_{\text {minus }}\right)} * 100(\%)$. Consequently, the power consumption can be saved as follows : $\frac{T_{\text {zero }}}{\left(T_{\text {plus }}+T_{\text {zero }}+T_{\text {minus }}\right)} * 100(\%)$.

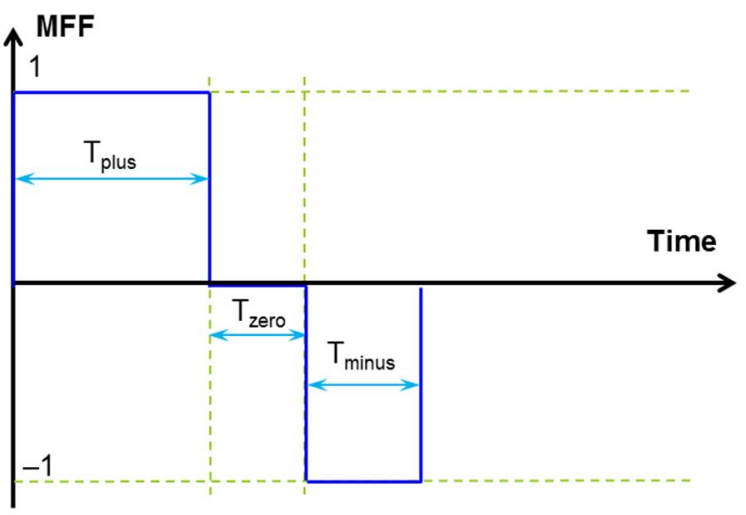

Fig. 8. Waveform of the modified field function.

\section{B. Numerical Simulation Studies}

With the same conditions as in the subsection III.C, we simulate to choose the parameters of the MFF. The positive and negative times are selected based on the guideline in condition 1 and 2. The zero time is chosen based on the following discussion: The zero time must be long enough for all the particles in the safe zone to reach to the correct outlet (except for the particles stuck at the vessel walls).

TABLE III. SimUlation RESUltS OF THE PARTICLE'S SUCCESS Ratios to THE CORRECT OUTLET WITH DIFFERENT VALUES OF $\mathrm{T}_{\text {MINUS }}$ AND $\mathrm{T}_{\text {ZERO }}$ WITH TPLUS $=8.5 \mathrm{~S}$

\begin{tabular}{|c|c|c|c|c|c|c|c|}
\hline $\begin{array}{c}\boldsymbol{T}_{\text {plus }}(\mathbf{s}) \\
\boldsymbol{T}_{\text {minus }}(\mathbf{s})\end{array}$ & 0 & 0.1 & 0.2 & 0.3 & 0.4 & 0.5 & 1 \\
\hline 5 & $99.9 \%$ & $100 \%$ & $100 \%$ & $100 \%$ & $100 \%$ & $100 \%$ & $100 \%$ \\
\hline 5.5 & $99.9 \%$ & $100 \%$ & $100 \%$ & $100 \%$ & $100 \%$ & $100 \%$ & $100 \%$ \\
\hline 5.6 & $99.9 \%$ & $99.9 \%$ & $100 \%$ & $100 \%$ & $100 \%$ & $100 \%$ & $100 \%$ \\
\hline 5.7 & $99.9 \%$ & $99.9 \%$ & $100 \%$ & $100 \%$ & $100 \%$ & $100 \%$ & $100 \%$ \\
\hline 5.8 & $99.9 \%$ & $99.9 \%$ & $100 \%$ & $100 \%$ & $100 \%$ & $100 \%$ & $100 \%$ \\
\hline 5.9 & $99.8 \%$ & $99.9 \%$ & $99.9 \%$ & $99.9 \%$ & $99.9 \%$ & $99.9 \%$ & $99.9 \%$ \\
\hline
\end{tabular}

We consider that $T_{\text {plus }}=8.5 \mathrm{~s}$ is the optimal value for the positive time. We increase the negative time as much as possible to reduce the heating of the coil that produce the 
positive magnetic field gradient. Table III presents the simulation results of the particle's success ratios to the correct outlet with different value of $\mathrm{T}_{\text {minus }}$ and $\mathrm{T}_{\text {zero. }}$. The second column in Table III is the results associated with original FF $\left(\mathrm{T}_{\text {zero }}=0\right)$, other columns are the results with MFF. Fig. 9.a and Fig. 9.b present the particle trajectory for $\mathrm{T}_{\text {zero }}=1 \mathrm{~s}, \mathrm{~T}_{\text {minus }}$ $=5.9 \mathrm{~s}$ and $\mathrm{T}_{\text {zero }}=0.2 \mathrm{~s}, \mathrm{~T}_{\text {minus }}=5.8 \mathrm{~s}$, respectively. With the MFF, the negative time can be increased to $5.8 \mathrm{~s}$. In this case, the minimum zero time is $0.2 \mathrm{~s}$. We can choose the zero time at any value if it is bigger than 0.2 . For example, if we choose zero time is $3 \mathrm{~s}$. Then the power consumption can be reduced to $\frac{3}{8.5+3+5.8} * 100=17.3(\%)$.

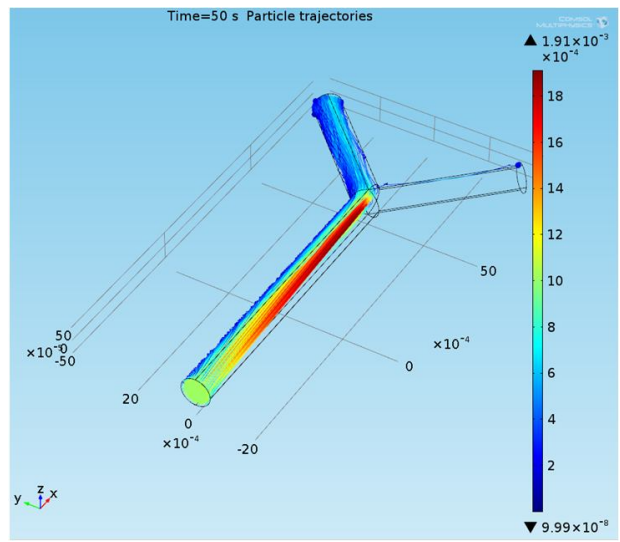

Fig. 9.a. Particle trajectories for 1000 particles with $\mathrm{T}_{\text {zero }}=1 \mathrm{~s}, \mathrm{~T}_{\text {minus }}=5.9 \mathrm{~s}$. Colors show the particles velocity $(\mathrm{mm} / \mathrm{s})$.

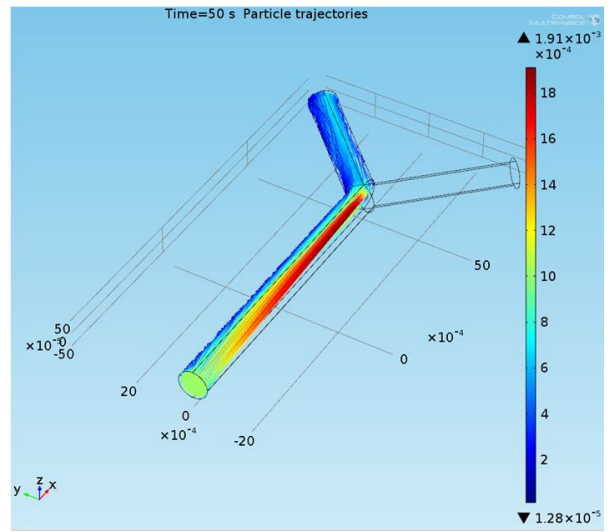

Fig. 9.b. Particle trajectories for 1000 particles with $\mathrm{T}_{\text {plus }}=0.2 \mathrm{~s}, \mathrm{~T}_{\text {minus }}=5.8 \mathrm{~s}$. Colors show the particles velocity $(\mathrm{mm} / \mathrm{s})$.

\section{CONClusion}

This paper presented the guidelines to select the optimal values of parameters for field function. Based on extensive simulation studies, the proposed guidelines were verified. Also, a modified field function concept was proposed to further optimize the MNPs' guidance performance with additional considerations of the operating conditions. By inserting a no-power time period, the power consumption as well as the heating could be significantly reduced in an electromagnetic actuator. Numerous simulation results were performed to prove the advantage of the proposed MFF scheme. In the future, we will experimentally prove the proposed design guidelines and the MFF scheme.

\section{REFERENCES}

[1] J. Bar, R. S. Herbst, and A. Onn, "Targeted drug delivery strategies to treat lung metastasis," Expert Opin. Drug Deliv., vol. 6, no. 10, pp. 1003-16, Oct. 2009.

[2] V. P. Torchilin, "Passive and active drug targeting: drug delivery to tumors as an example," Handb. Exp. Pharmacol., no. 197, pp. 3-53, Jan. 2010.

[3] S. Martel, "Magnetic nanoparticles in medical nanorobotics," $J$. Nanopart. Res., vol. 17, no. 2, pp. 75, Feb. 2015.

[4] M. Hamdi and A. Ferreira, "Guidelines for the design of magnetic nanorobots to cross the blood-brain barrier," IEEE Trans. Robot., vol. 30, no. 1, pp. 81-92, Feb. 2014.

[5] B. Gleich, N. Hellwig, H. Bridell, R. Jurgons, C. Seliger, C. Alexiou, B. Wolf, and T. Weyh, "Design and Evaluation of Magnetic Fields for Nanoparticle Drug Targeting in Cancer," IEEE Trans. Nanotechnol., vol. 6, no. 2, pp. 164-170, Mar. 2007.

[6] E. M. Cherry, P. G. Maxim, and J. K. Eaton, "Particle size, magnetic field, and blood velocity effects on particle retention in magnetic drug targeting," Med. Phys., vol. 37, no. 1, p. 175, 2010.

[7] A. E. David, A. J. Cole, B. Chertok, Y. S. Park, and V. C. Yang, "A combined theoretical and in vitro modeling approach for predicting the magnetic capture and retention of magnetic nanoparticles in vivo," $J$. Control. Release, vol. 152, no. 1, pp. 67-75, May 2011.

[8] A. Nacev, C. Beni, O. Bruno, and B. Shapiro, "The Behaviors of Ferro-Magnetic Nano-Particles In and Around Blood Vessels under Applied Magnetic Fields," J. Magn. Magn. Mater., vol. 323, no. 6, pp. 651-668, Mar. 2011.

[9] S. Kenjereš and B. W. Righolt, "Simulations of magnetic capturing of drug carriers in the brain vascular system," Int. J. Heat Fluid Flow, vol. 35, pp. 68-75, Jun. 2012.

[10] M. Larimi, A. Ramiar, and A. Ranjbar, "Numerical simulation of magnetic nanoparticles targeting in a bifurcation vessel," J. Magn. Magn. Mater., vol. 362, pp. 58-71, Aug. 2014.

[11] B. Chertok, A. E. David, and V. C. Yang, "Brain tumor targeting of magnetic nanoparticles for potential drug delivery: effect of administration route and magnetic field topography," J. Control. Release, vol. 155, no. 3, pp. 393-9, Nov. 2011.

[12] P. Vartholomeos and C. Mavroidis, "In silico studies of magnetic microparticle aggregations in fluid environments for MRI-guided drug delivery," IEEE Trans. Biomed. Eng., vol. 59, no. 11, pp. 3028-38, Nov. 2012.

[13] M. Dadkhah Tehrani and J. Yoon, "Statistical investigation of efficiency of the nanomagnetic particle steering in blood vessels," in the 7th IEEE Int. Conf. on Nano/Molecular Medicine and Engineering, 2013, pp. 36-40.

[14] M. D. Tehrani, J. H. Yoon, M. O. Kim, and J. Yoon, “ A novel scheme for nanoparticle steering in blood vessels using a functionalized magnetic field," IEEE Trans. Biomed. Eng., vol. 62, no. 1, pp. 303-313, Jan. 2015.

[15] S. Einav, D. Elad, "Physical and flow properties of blood," in Biomedical Engineering and Design Handbook, vol. 1, M. Kutz, Ed. McGraw Hill Professional, Access Engineering, 2009, pp. 3.1-3.25.

[16] H. Cao, G. Huang, S. Xuan, Q. Wu, F. Gu, and C. Li, "Synthesis and characterization of carbon-coated iron core/shell nanostructures," $J$. Alloys Compd., vol. 448, no. 1-2, pp. 272-276, Jan. 2008. 